\title{
Hepatocyte growth factor activator inhibitors (HAI-1 and HAI-2) are potential targets in uterine leiomyosarcoma
}

\author{
KEIICHIRO NAKAMURA ${ }^{1}$, FERNANDO ABARZUA ${ }^{2}$, ATSUSHI HONGO ${ }^{1}$, JUNICHI KODAMA ${ }^{1}$, \\ YASUTOMO NASU $^{2}$, HIROMI KUMON ${ }^{2}$ and YUJI HIRAMATSU ${ }^{1}$ \\ ${ }^{1}$ Department of Obstetrics and Gynecology, Okayama University Graduate School of Medicine, \\ ${ }^{2}$ Dentistry and Pharmaceutical Sciences, 2-5-1 Shikata-cho, Kita-ku, Okayama 700-8558, Japan
}

Received March 31,2010; Accepted May 21, 2010

DOI: 10.3892/ijo_00000709

\begin{abstract}
Hepatocyte growth factor activator inhibitors (HAI-1 and HAI-2) are Kunitz-type serine protease inhibitors that have a broad inhibitory spectrum against serine proteases. This study examined the role of HAI-1 and HAI-2 in uterine leiomyosarcoma (LMS) patients, and in vitro. HAI-1 and HAI-2 was examined in uterine normal smooth muscle, leiomyoma and LMS specimens using immunohistochemistry. We investigated biological functions and inhibitory effects of HAI-1 and HAI-2 using uterine LMS cell line SK-LMS-1 and SKN. The expression levels of HAI-1 and HAI-2 were significantly decreased in uterine LMS specimens relative to corresponding uterine normal smooth muscle and leiomyoma specimens. Furthermore, the low HAI-1 and HAI-2 expression was a significant predictor for poor prognosis when compared with high HAI-1 and HAI-2 expression (disease-free survival rate; $\mathrm{p}=0.024$ and $\mathrm{p}=0.045$, overall survival rate; $\mathrm{p}=0.043$ and $\mathrm{p}=0.009)$. HAI- 1 and HAI-2 showed potential inhibitory effects that mediated cell proliferation, migration and cellular invasion which led to apoptosis and necrosis through a reduction of HGFA, matriptase and hepsin expression. These findings indicate that HAI-1 and HAI-2 may be possible tumor suppressor genes for uterine LMS and thus, both could be considered therapeutic agents for the treatment of LMS.
\end{abstract}

\section{Introduction}

The incidence of uterine cancer throughout the world has markedly increased in recent years. This has been related, in part, to an increase in uterine sarcomas a relatively rare type,

Correspondence to: Dr Keiichiro Nakamura, Department of Obstetrics and Gynecology, Okayama University Graduate School of Medicine, Dentistry and Pharmaceutical Sciences, 2-5-1 Shikatacho, Kita-ku, Okayama 700-8558, Japan

E-mail: k-nakamu@cc.okayama-u.ac.jp

Key words: uterine leiomyosarcoma, HAI-1, HAI-2, tumor suppressor gene accounting for $3-5 \%$ of all uterine cancers $(1,2)$. Uterine sarcomas have been classified into three main histologic subgroups, in order of decreasing incidence: carcinosarcoma, leiomyosarcoma (LMS) and endometrial stromal sarcoma. Each group of tumors is distinct with respect to pattern spread, pathological features, prognostic factors and response to treatment $(3,4)$. Uterine LMS is a malignant tumor composed of cells showing distinct features of the smooth muscle lineage. Uterine LMS is graded according to a modified International Federation of Gynecology and Obstetrics (FIGO) staging system for endometrial cancer (5). Although complete resection is frequently accomplished, the risk for recurrence after complete resection of FIGO stage I or II high grade uterine LMS is $50-80 \%$ at 2 years (6-9). The risk of recurrence is greater for patients with high stage disease, and is likely greater for tumors with higher mitotic rates, although no standard prognostic criteria have been established $(3,10)$. It is now widely accepted that new approaches for the treatment of uterine LMS are pivotal to further improve the prognosis of the disease.

The malignant process involves degradation of the extracellular matrix (ECM), including the interstitial basement membranes by proteinases, which facilitates cell detachment followed by local and systemic spreading. Hepatocyte growth factor (HGF) is a multifunctional growth factor that is secreted by mesenchymal cells in the liver as an inactive single-chain pro-peptide and normally remains in this form associated with the ECM (11). HGF is a mesenchymal-derived cytokine that acts on motility and morphogenesis in various target cells. The pleiotropic activities of HGF are mediated through its receptor, a transmembrane tyrosine kinase encoded by the pro-oncogene c-Met $(12,13)$. Aberrant HGF/c-Met expressions have been implicated in human uterine LMS (14). HGF/c-Met is secreted as an inactive pro-peptide which is cleaved by HGF activator (HGFA) into its active form. HGFA is regulated by two inhibitors, HGFA inhibitor type I (HAI-1) and type II (HAI-2), both are a type 1 transmembrane protein with $\mathrm{NH}_{2}$-terminal Kunitz domain (KD1) and $\mathrm{COOH}$-terminal KD (KD2) in their extracellular portion (15-18). HAI-1 and HAI-2 potently inhibit a variety of enzyme serine proteases that may be involved in carcinogenesis, invasion and metastasis. HAI-1 and 
HAI-2 appear to be the cognate inhibitors of HGFA, matriptase, hepsin and prostasin. Among them, hepsin and matriptase belong to the type II transmembrane serine protease superfamily, whereas prostasin is a glycosylphosphatidylinositol-anchored membrane serine protease protein. Hepsin, matriptase and prostasin are potential candidates for the cellular activities of pro-hepatocyte growth factor (pro-HGF) in cancer tissues (19-21). HAI-2 is a more efficient inhibitor of hepsin (22), and displays a broader inhibitory spectrum than HAI-1 $(16,23)$. To date, several studies on HAI-1 and HAI-2 expression in tumor tissues have been published. Previously, we reported that HAI-1 and HAI-2 levels are significantly decreased during the progression of cervical and ovarian cancer (24-26). It has been reported that the reduced expression of HAI-1 is possibly involved in the progression of prostate, breast, and gastric cancer (27-29). Several studies have shown that the down-regulation of HAI-2 in glioblastomas, hepatocellular and renal carcinomas was partly due to the hypermethylation of the HAI-2 promoter region (30-32). However, it is not clear how the mechanisms of action of HAI-1 and HAI-2 are involved in uterine LMS, or how their roles are intertwined. In this study, we examined the functional role of HAI -1 and HAI-2 in uterine LMS patients and in vitro.

\section{Patients and methods}

Patients and tissues. Patients with uterine normal smooth muscle $(n=15)$, leiomyoma $(n=15)$ and LMS $(n=12)$ were treated at Okayama University Hospital between January 1996 and December 2008. Each of these patients underwent abdominal total hysterectomy at the Department of Obstetrics and Gynecology of Okayama University Hospital. Tumor specimens were obtained at the time of surgery and immediately fixed in $10 \%$ neural-buffered formalin, and embedded in paraffin. Surgical staging was reviewed based on the FIGO staging system: eight were allocated to stage I, three to stage II, and one to stage III. The disease-free survival (DFS) and overall survival (OS) rates were defined as the interval from the initial surgery to clinically proven recurrence and death, respectively. The end date of the follow-up study for conducting the analysis was 30th June 2009.

Immunohistochemical analysis and staining evaluation. Formalin-fixed, paraffin-embedded sections, at $4 \mu \mathrm{m}$ thick, were deparaffinized with xylene and re-hydrated in ethanol. Endogenous peroxidase activity was quenched by methanol containing $0.3 \%$ hydrogen peroxidase for $15 \mathrm{~min}$. Then, the sections were incubated at room temperature with a primary antibody for HAI-1 and HAI-2 (Santa Cruz Biotechnology, Santa Cruz, CA) followed by staining using a streptavidinbiotin-peroxidase kit (Nichirei, Tokyo, Japan). The sections were counterstained with hematoxylin. The level of staining in the tumor was expressed by classifying into fourth groups by scoring the percentage of positive stained cells: 0 (negative, $<10 \%$ stained cells), $1+$ (weak, $10-25 \%$ stained cells), $2+$ (moderate, $26-50 \%$ stained cells) and $3+$ (strong, $>50 \%$ stained cells). Two independent examiners with no prior knowledge of the patients' clinical data conducted the microscopic evaluations. Controversial cases were evaluated during a microscope conference.
Cell culture, media, and generation of transfectants. SK-LMS-1 (ATCC no. HTB-88) and SKN [Japanese Collection of Research Bioresources (JCRB) no. IFO50314] cell lines were derived from human LMSs. The SK-LMS-1 cell line was maintained in Dulbecco's modified Eagle's medium (DMEM) (Life Technologies, Grand Island, NY), supplemented with $10 \%$ fetal bovine serum (FBS). The SKN cell line was maintained in Ham's F12 medium with $10 \%$ FBS. Cells were grown at $37^{\circ} \mathrm{C}$ in an atmosphere with $5 \% \mathrm{CO}_{2}$ in air. A cDNA encoding the whole coding region of HAI-1 or HAI-2 was constructed by polymerase chain reaction using full-length HAI-1 cDNA or HAI-2 cDNA as a template. The polymerase chain reaction products were subcloned into the EcoRI/SalI site (HAI-1) or the XbaI/SalI site (HAI-2) of the expression plasmid pCIneo (Promega, Madison, WI), as described previously (33). The HAI-1 and HAI-2 cDNA expression vectors were transfected into each cell line using the TransFast transfection reagent (Promega). Mock transfected cells served as a control.

Western blot analysis. Cell lysates were collected and estimated using a protein assay system (Bio-Rad, Hercules, CA) according to the manufacturer's protocols. Proteins from each cell line were subjected to SDS-PAGE, and were transferred onto a nitrocellulose membrane. Polyclonal and monoclonal antibodies used for immunoblotting were as follows: HAI-1, HAI-2, and B-actin (Sigma Chemical Co., St. Louis, MO). The working dilution of all primary antibodies was 1:1000. Membranes were then incubated with the appropriate secondary antibodies. Expressions of antigen-antibody complexes were detected with an enhanced chemiluminescence kit (Amershan Biosciences, Piscataway, NJ).

MTS assay and cell viability assay. In order to evaluate the effects of HAI-1 and/or HAI-2 on cell proliferation, the MTS assay (Promega) was performed. Cells were seeded into 96-well plates and transfected when the cell density reached $5 \times 10^{4}$ cells/well. Cells were transiently transfected with either HAI-1 vector or HAI-2 vector, or both HAI-1 vector and HAI-2 vector for 12, 24, 36 and $48 \mathrm{~h}$ and then MTS was added for $1 \mathrm{~h}$. The absorbance was read at a wavelength of $490 \mathrm{~nm}$ using an ELISA plate-reader (Bio-Rad Systems). In order to examine the cell viability after transient transfection with either HAI-1 or HAI-2, or both HAI-1 and HAI-2 in SK-LMS-1 and SKN cell lines, SYTO 10 green fluorescent nucleic acid stain and Dead red (ethidium homodimer-2) nucleic acid stain $\left(\right.$ Live/Dead ${ }^{\circledR}$ ) reduced biohazard viability/cytotoxicity kit; Invitrogen, Eugene, OR) were added to each well and incubated for $15 \mathrm{~min}$. Cell fluorescence was observed using a fluorescent microscope (Olympus, Tokyo, Japan).

Motility invasion assay. For the evaluation of motility of SK-LMS-1 and SKN cells, a monolayer wounding (scratch) assay was performed. Cells were allowed to form a monolayer on a culture dish, and a wound was made by scratching the monolayer with a pipette tip. After the scratched cells were removed and transiently transfected with either HAI-1 vector or HAI-2 vector, or both HAI-1 vector and HAI-2 vector, the cells were cultivated for $12 \mathrm{~h}$. The cellular motility was also estimated using Chemotaxi-cells (polycarbonate filter, 
pore size $8 \mu \mathrm{m}$; Kurabo, Osaka, Japan) coated with type IV collagen $\left(3.6 \mu \mathrm{g}\right.$ per filter). Cells $\left(1 \times 10^{5}\right)$ in $100 \mu \mathrm{l}$ of DMEM or Ham's F12 medium and $0.1 \%$ bovine serum albumin were placed in the upper compartment and incubated for $24 \mathrm{~h}$. After incubation, the cells on the upper surface of the filter were wiped off with a cotton swab. The cells on the lower surface were stained with hematoxylin and counted in ten randomly selected fields.

Matrigel invasion assay. To investigate differences in the matrigel invasive ability between cells expressing HAI-1 and HAI-2, we used the BD BioCoat Matrigel Invasion Chamber (BD Bioscience, Bedford, MA). The SK-LMS-1 and SKN cells transiently transfected with either HAI-1 vector or HAI-2 vector, or both HAI-1 vector and HAI-2 vector were added in situ with $10 \mu \mathrm{g} / \mathrm{ml}$ of DiI (Invitrogen, Carlsbad, CA) in DMEM or Ham's F12 with $10 \%$ FBS for $1 \mathrm{~h}$. Cells $\left(5 \times 10^{4}\right)$ of each genotype were added to inserts, and $0.75 \mathrm{ml}$ of medium was added to the bottom of each well. After $48 \mathrm{~h}$ of incubation, membranes were removed from the insert and mounted on slides, and then invading cells were counted under the microscope. Matrigel assays were performed in triplicate.

Reverse transcription (RT)-PCR and real-time RT-PCR. Total RNA was extracted from cell lines using the acid guanidiumphenol-chloroform method (Isogen, Nippon Gene, Tokyo, Japan) according to the manufacturer's instructions. The PCR products were analyzed using $1.5 \%$ agarose gel electrophoresis. For quantitative real-time RT-PCR using cyber green, PCR was performed in a Light Cycler (Roche Applied Science, Mannheim, Germany). As the internal control, glyceraldehyde3-phosphate dehydrogenase (GAPDH) mRNA was also measured using RT-PCR and real-time PCR. The primers for HAI-1, HAI-2, HGFA, matriptase, hepsin, prostasin, GAPDH, were as described previously $(28,34-36)$.

FITC-conjugated Annexin V assay. In order to evaluate the effects of HAI-1 and/or HAI-2 on apoptosis and necrosis, an Annexin V-FITC reagent assay (BioVision, Mountain View, CA) was performed. Cells were seeded into 96-well plates and transfected when the cell density reached $5 \times 10^{4}$ cells/well. Cells were transiently transfected with either HAI-1 vector or HAI-2 vector, or both HAI-1 vector and HAI-2 vector for $48 \mathrm{~h}$ and then the cells were removed. A total of $2 \times 10^{5}$ cells were collected by centrifugation. Apoptosis was measured by staining with FITC-conjugated Annexin V coupled with flow cytometric analysis according to the manufacturer's recommendations.

Cell growth in monolayer, cell aggregation and soft agar. For evaluation of cell growth in a monolayer, cells were plated at a density of $3 \times 10^{4}$ cells/well in 6-well plates containing DMEM or Ham's F12 with 10\% FBS. The cell number was counted in triplicate after 1, 3, 5 and 7 days using hemocytometer to assess cell proliferation. To evaluate growth during cell aggregation, a cell suspension $\left(1 \times 10^{4}\right.$ cells/well $)$ in $1 \mathrm{ml} \mathrm{DMEM}$ or Ham's F12 with $10 \%$ FBS was overlaid onto a 35 -mm dish containing a $0.5 \%$-agar base. Cells were observed under a microscope (Olympus) on day 10 after culture. To evaluate growth in soft agar, a cell suspension (1x $10^{4}$ cells/well) in $1 \mathrm{ml} 0.2 \%$ Noble agar DMEM or Ham's F12 with $10 \%$ FBS was overlaid onto a $35-\mathrm{mm}$ dish containing a $0.5 \%$-agar base. Colonies $>0.2 \mathrm{~mm}$ in diameter were counted on day 21 after culture. Soft agar assays were performed in triplicate.

Statistical analysis. Statistical analyses were performed using the Mann-Whitney U test for comparison with the control and One-factor ANOVA followed by Fisher's protected least significance difference test for all pair wise comparisons. The analyses were performed with the software package StatView version 5.0 (Abacus Concepts, Berkeley, CA). Differences were considered significant at $\mathrm{p}<0.05$.

\section{Results}

Immunoassays. HGF-dependent activation may have a role in conventional LMS tumorigenesis, thus the pro-HGF-activation machinery might be important. However, the role of HAI-1 and HAI-2 in uterine LMS is not known. Fig. 1A1-4 illustrates the representative immunoassaying patterns of HAI-1 and HAI-2. HAI-1 and HAI-2 expression levels were significantly decreased in uterine LMS specimens relative to the corresponding uterine normal smooth muscle and leiomyoma specimens (Fig. 1B). Furthermore, the DFS and OS curves of the 12 LMS patients according to their HAI-1 and HAI-2 expression status are shown in Fig. 1C-F, respectively. The DFS and OS rates of patients exhibiting high HAI-1 and HAI-2 expression were significantly higher than those of patients exhibiting low HAI-1 and HAI-2 expression (DFS; $\mathrm{p}=0.024$ and $\mathrm{p}=0.045, \mathrm{OS} ; \mathrm{p}=0.043$ and $\mathrm{p}=0.009$ ), respectively, Mann-Whitney U test).

The protein expression and cell proliferation. The purpose of this experiment was to examine the functional role of HAI-1 and HAI-2. A significant increase in expression of HAI-1 and HAI-2 resulted when the cells were transiently transfected with either HAI-1 vector or HAI-2 vector, or both HAI-1 vector and HAI-2 vector (Fig. 2A). The cell proliferation of SK-LMS-1 and SKN cells transiently transfected with either HAI-1 vector or HAI-2 vector, or both HAI-1 vector and HAI-2 vector was significantly inhibited as evaluated by the MTS assay (Fig. 2B). Cell viabilities of the SK-LMS-1 and SKN cell lines were evaluated after transient transfection with either HAI-1 vector or HAI-2 vector, or both HAI-1 vector and HAI-2 vector. The percentages of viable cells were decreased to 77.2, 72.7 and $68.1 \%$ (SK-LMS-1), and 73.0, 69.2 and $66.1 \%$ (SKN) of the control cell viabilities at $48 \mathrm{~h}$ after transient transfection with either HAI-1 vector or HAI-2 vector, or both HAI-1 vector and HAI-2 vector, respectively (Fig. 2C).

Motility invasiveness and matrigel invasion assessment. The purpose of these experiments was to study the role of uterine LMS in the motility invasiveness and matrigel invasion of cells transiently transfected with either HAI-1 vector or HAI-2 vector, or both HAI-1 vector and HAI-2 vector. The cells transiently transfected with either HAI-1 vector or HAI-2 vector, or both HAI-1 vector and HAI-2 vector were much slower as compared with the control on motility invasiveness 
A

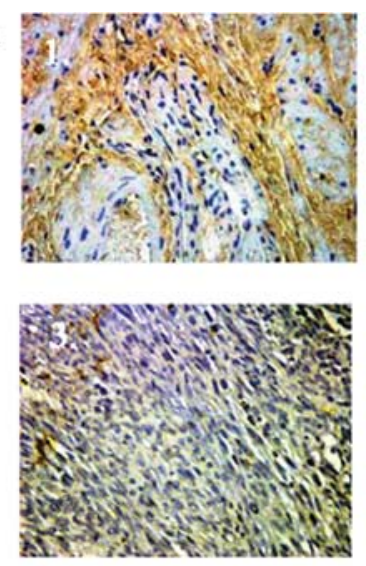

C

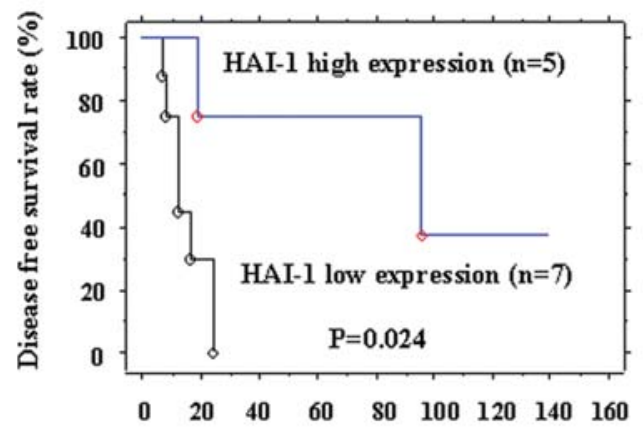

$\mathbf{E}$

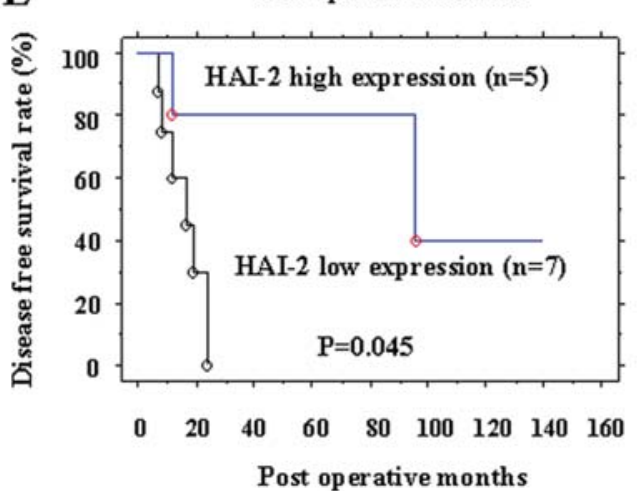

B

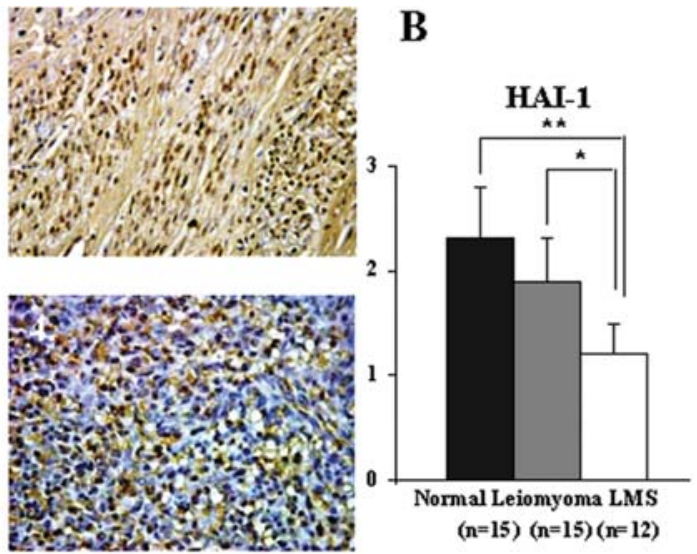

D

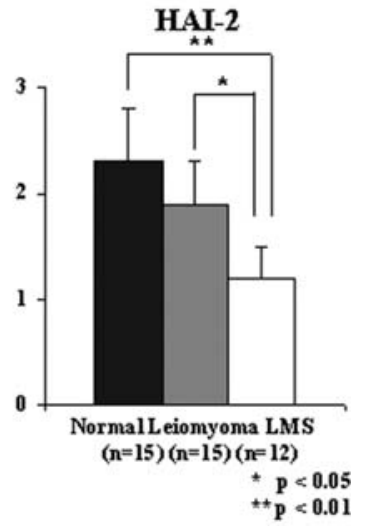

F

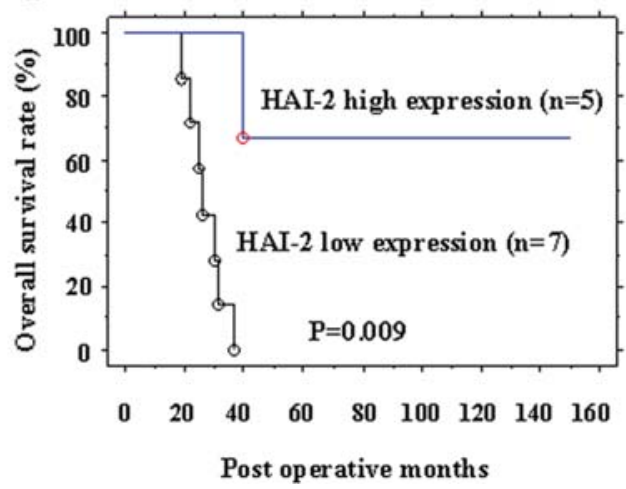

Figure 1. Representative immunostaining patterns of hepatocyte growth factor activator inhibitors (HAI-1 and HAI-2). (A) 1, moderate HAI-1 staining in uterine leiomyoma; 2, strong HAI-2 staining in uterine normal smooth muscle; 3, negative HAI-2 staining in uterine leiomyosarcoma (LMS); 4, weak HAI-1 staining in uterine LMS (original magnification x200). (B) histogram of HAI-1 and HAI-2 expression by specimens (uterine normal smooth muscle, leiomyoma and LMS). Kaplan-Meier plots for: (C) disease-free; and (D) overall survival of the 12 patients with LMS according to HAI-1 expression status. Kaplan-Meier plots for: (E) disease-free; and (F) overall survival of the 12 patients with LMS according to HAI-2 expression status. Low epithelial expression, score $0-1$; high epithelial expression, score 2 and 3.

(Fig. 3A and B). These results suggest that HAI-1 and HAI-2 overexpression decreases cell adhesion and spreading. The percentage of cells reaching the bottom of the filter was decreased to 69.8 and $64.8 \%, 54.3$ and $49.7 \%, 52.8$ and $46.6 \%$ on matrigel invasion at $48 \mathrm{~h}$ after transient transfection with either HAI-1 vector or HAI-2 vector, or both HAI-1 vector and HAI-2 vector into SK-LMS-1 and SKN cell lines, respectively (Fig. 3C).

$R T-P C R$ and real-time RT-PCR. The transient transfection with either HAI-1 vector or HAI-2 vector, or both HAI-1 vector and HAI-2 vector into SK-LMS-1 and SKN cell lines caused a significant increase in expression of HAI-1 and
HAI-2. The expression of HGFA, matriptase and hepsin in SK-LMS-1 and SKN cells was decreased by transient transfection with either HAI-1 vector or HAI-2 vector, or both HAI-1 and HAI-2 vector. However, the expression of prostasin was not related to the transient transfection with either HAI-1 vector or HAI-2 vector, or both HAI-1 vector and HAI-2 vector (Fig. 4).

FITC-conjugated Annexin $V$ assay. The representative flow cytometric data revealed that transient transfection with either HAI-1 vector or HAI-2 vector, or both HAI-1 vector and HAI-2 vector for $48 \mathrm{~h}$ resulted in increased FITC positive and propidium iodide positive signals. HAI-1 and 
A

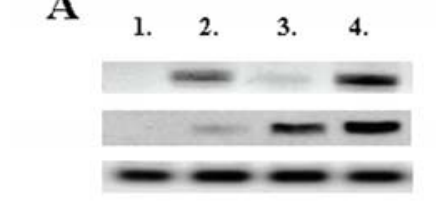

5. 6.7 .8 .

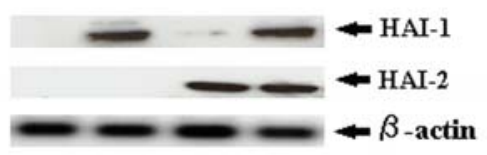

1. SK-LMS-1 Empty Vector

2. SK-LMS-1 HAI-1

3. SK-LMS-1 HAI-2

4. SK-LMS-1 HAI-1+HAI-2

5. SKN Empty Vector

6. SKN HAI-1

B

8. SKN HAI-1+HAI-2

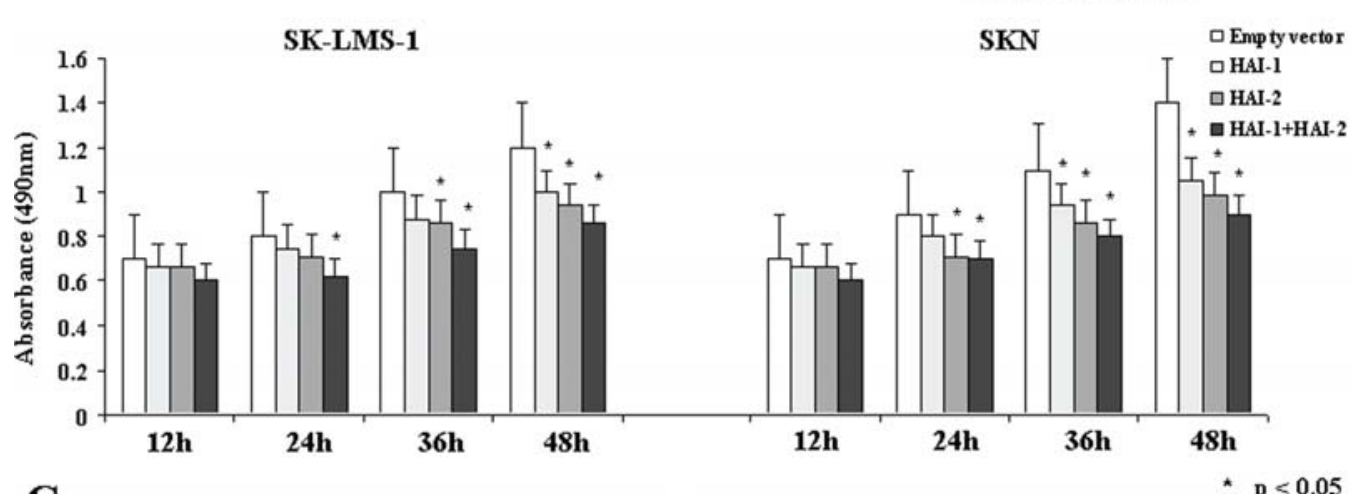

C
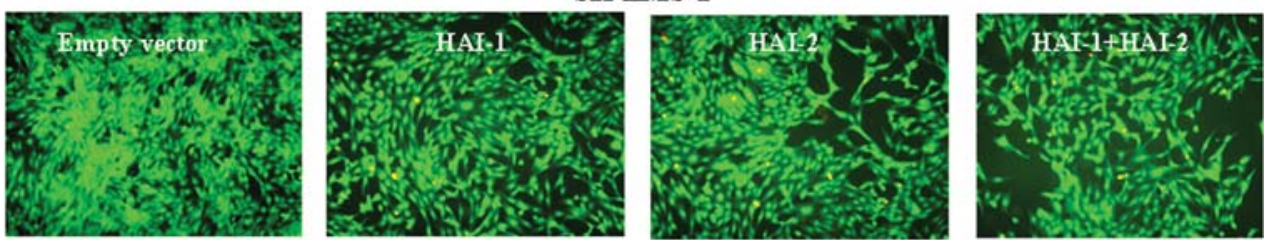

SKN
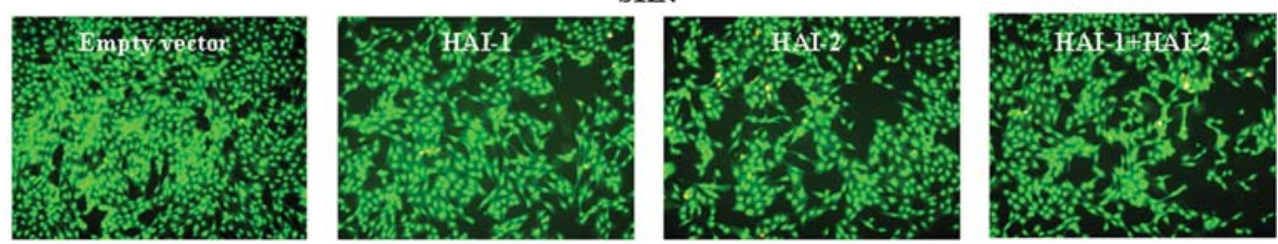

Figure 2. Evaluation of cells transiently transfected with either HAI-1 vector or HAI-2 vector, or both HAI-1 vector and HAI-2 vector. (A) Western blot analysis of HAI-1 and HAI-2 expression after transient transfection with either HAI-1 vector or HAI-2 vector, or both HAI-1 vector and HAI-2 vector in SK-LMS-1 and SKN cells for $48 \mathrm{~h}$. B-actin antibody was used as the loading control in the same blot. (B) MTS assays of the cell proliferation of SK-LMS-1 and SKN cells after transient transfection with either HAI-1 vector or HAI-2 vector, or both HAI-1 vector and HAI-2 vector for $12,24,36$ and 48 h. The assays were carried out in quadruplicate transfection experiments. (C) The cell viabilities of SK-LMS-1 and SKN cells after transient transfection with either HAI-1 vector or HAI-2 vector, or both HAI-1 vector and HAI-2 vector evaluated using a fluorescence microscope.

HAI-2 induced both apoptosis and necrosis in these cancer cells, which was confirmed by the results of the Annexin V-FITC assay as shown in Fig. 5. Transfection with either HAI-1 vector or HAI-2 vector, or both HAI-1 vector and HAI-2 vector increased the total apoptotic and necrotic cells to $25.92,33.42$ and $36.93 \%$, and $39.11,55.32$ and $59.73 \%$ as compared with the control levels of 9.38 and $7.83 \%$ in SK-LMS-1 and SKN cells, respectively.

Inhibition of cell growth in a monolayer, cell aggregation, and anchorage-independent cell growth in soft agar. We found a significant inhibitory effect of HAI-1 and HAI-2 on monolayer cell growth in SK-LMS-1 and SKN cells transiently transfected with either HAI-1 vector or HAI-2 vector, or both HAI-1 vector and HAI-2 vector compared to the control (Fig. 6A). Furthermore, the transient transfection with either HAI-1 vector or HAI-2 vector, or both HAI-1 vector and HAI-2 vector into either cell line resulted in a significantly reduced number of colonies in cell aggregation tests and soft agar when compared to the control $(\mathrm{p}<0.01)$ (Fig. 6B and C).

\section{Discussion}

HGFA is responsible for the proteolytic activation of the precursor form of HGF in various human cancer tissues and sera $(22,28,37)$. To date, two specific inhibitors of HGFA have been identified, HAI-1 and HAI-2 are Kunitztype inhibitors that have a broad inhibitory spectrum against serine proteases. These unusual serine protease inhibitors are type I transmembrane glycoproteins that contain two extracellular Kunitz-type inhibitory domains (33). Our previous results indicated that reduced expression of HAI-1 and HAI-2 is possibly involved in disease progression in cervical and ovarian cancers (24-26). In the present study, we examined whether HAI-1 and HAI-2 expression were correlated with uterine normal smooth muscle, leiomyoma and LMS specimens. Szabo and colleagues have reported 
A
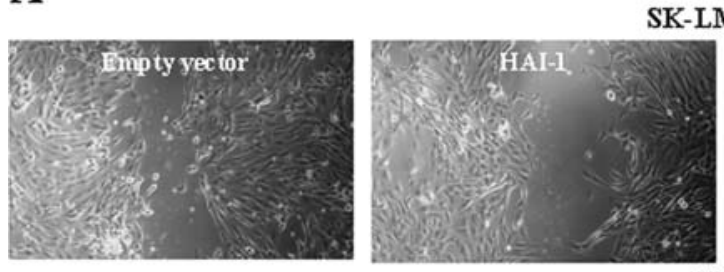

SK-LMS-1
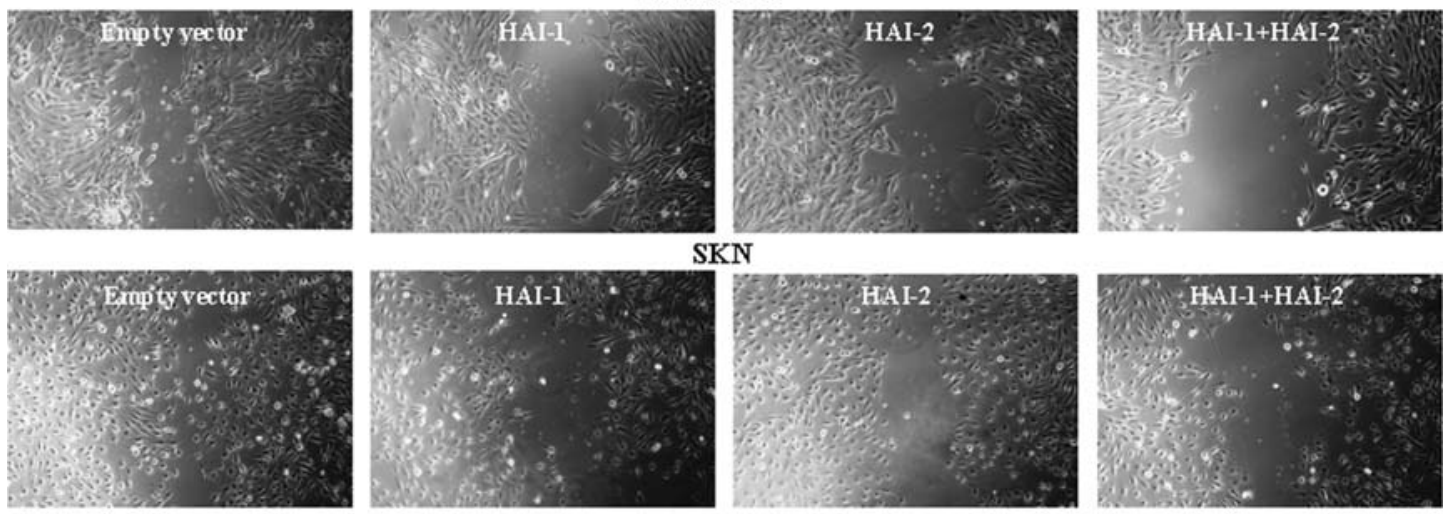

SKN

B Migration assay on type IV collagen
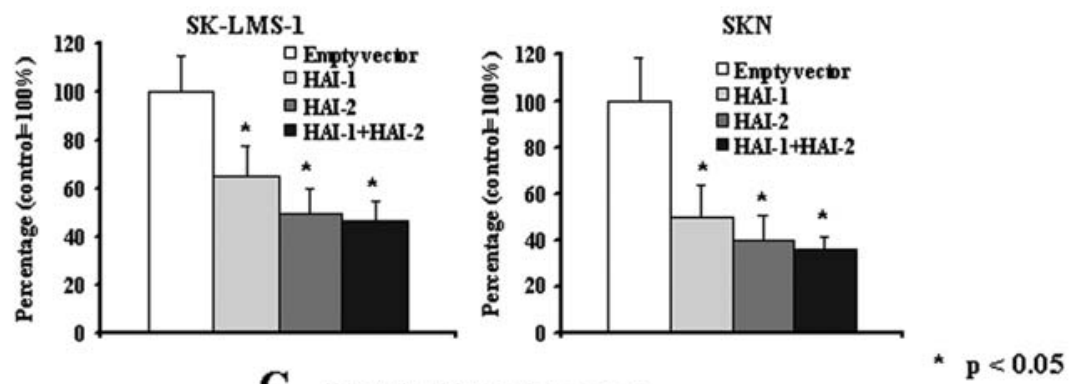

C Matrigel invasion assay SK-LMS-1
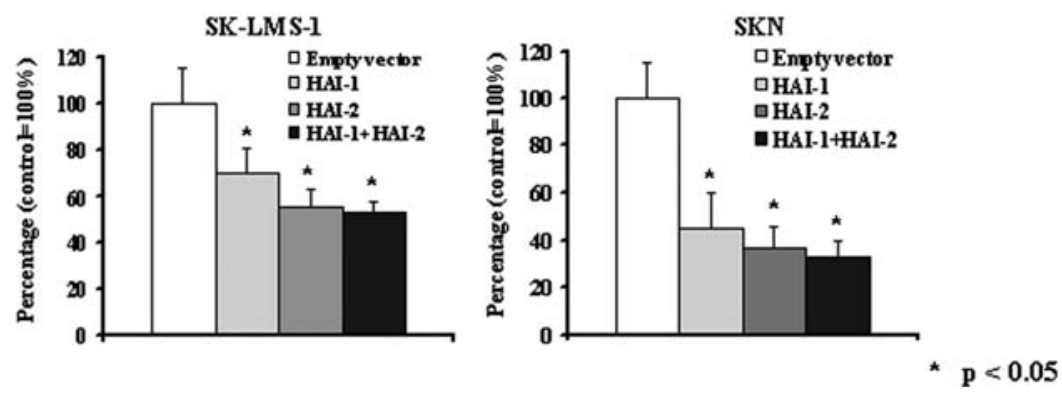

Figure 3. Effects on the motility and matrigel invasion of SK-LMS-1 and SKN cells after transient transfection with either HAI-1 vector or HAI-2 vector, or both HAI-1 vector and HAI-2 vector. (A) Cell scratch assay of SK-LMS-1 and SKN cells after transient transfection with either HAI-1 vector or HAI-2 vector, or both HAI-1 vector and HAI-2 vector for $12 \mathrm{~h}$. (B) Migration assay on type IV collagen of SK-LMS-1 and SKN cells after transient transfection with either HAI-1 vector or HAI-2 vector, or both HAI-1 vector and HAI-2 vector for $24 \mathrm{~h}$. (C) Matrigel invasion assay after transient transfection with either HAI-1 vector or HAI-2 vector, or both HAI-1 vector and HAI-2 vector into SK-LMS-1 and SKN cells for $48 \mathrm{~h}$. Following incubation, membranes were removed from the insert and mounted on slides. The numbers of invading cells were counted under the microscope. The motility and matrigel assays were performed in triplicate.

that studies demonstrating surface epithelium and uterine glands of the human normal uterus tract showed a high level of both HAI-1 and HAI-2 expressions (38). The levels of HAI-1 and HAI-2 expression were significantly decreased in uterine LMS specimens relative to corresponding uterine normal smooth muscle and leiomyoma specimens. Futhermore, the low levels of HAI-1 and HAI-2 expressions were significantly associated with a poor prognosis in uterine LMS. These findings indicate that HAI- 1 and HAI- 2 proteins could be important tumor suppressor genes for identifying uterine LMS.

HAI-1 and HAI-2 were originally described as endogenous inhibitors of HGFA, matriptase, hepsin, and prostasin (15-18). They both have a serine proteinase domain and show enhanced expression in a variety of tumor tissues (39).
Hepsin and matriptase have been proposed to initiate signaling and proteolytic cascades through their ability to activate pro-uPA, and matriptase is known to interact with prostasin (39-43). In this study, HGFA, matriptase, and hepsin were significantly inhibited by HAI-1 and HAI-2 in uterine LMS cell lines (Fig. 4A). These membrane-bound proteases are likely to have important roles in cellular homeostasis and their dysregulated activities and expression have been implicated in tumor development and progression. To date, several studies have suggested a possible role for HAI-1 and HAI-2 in the invasion of carcinoma cells. Our previous results also showed that HAI-1 and HAI-2 inhibited cell growth and promoted apoptosis in cervical and ovarian cancers (24-26). In breast cancer, inactivation of HAI-1 and its homologous protein HAI-2 significantly increased HGF mediated breast 


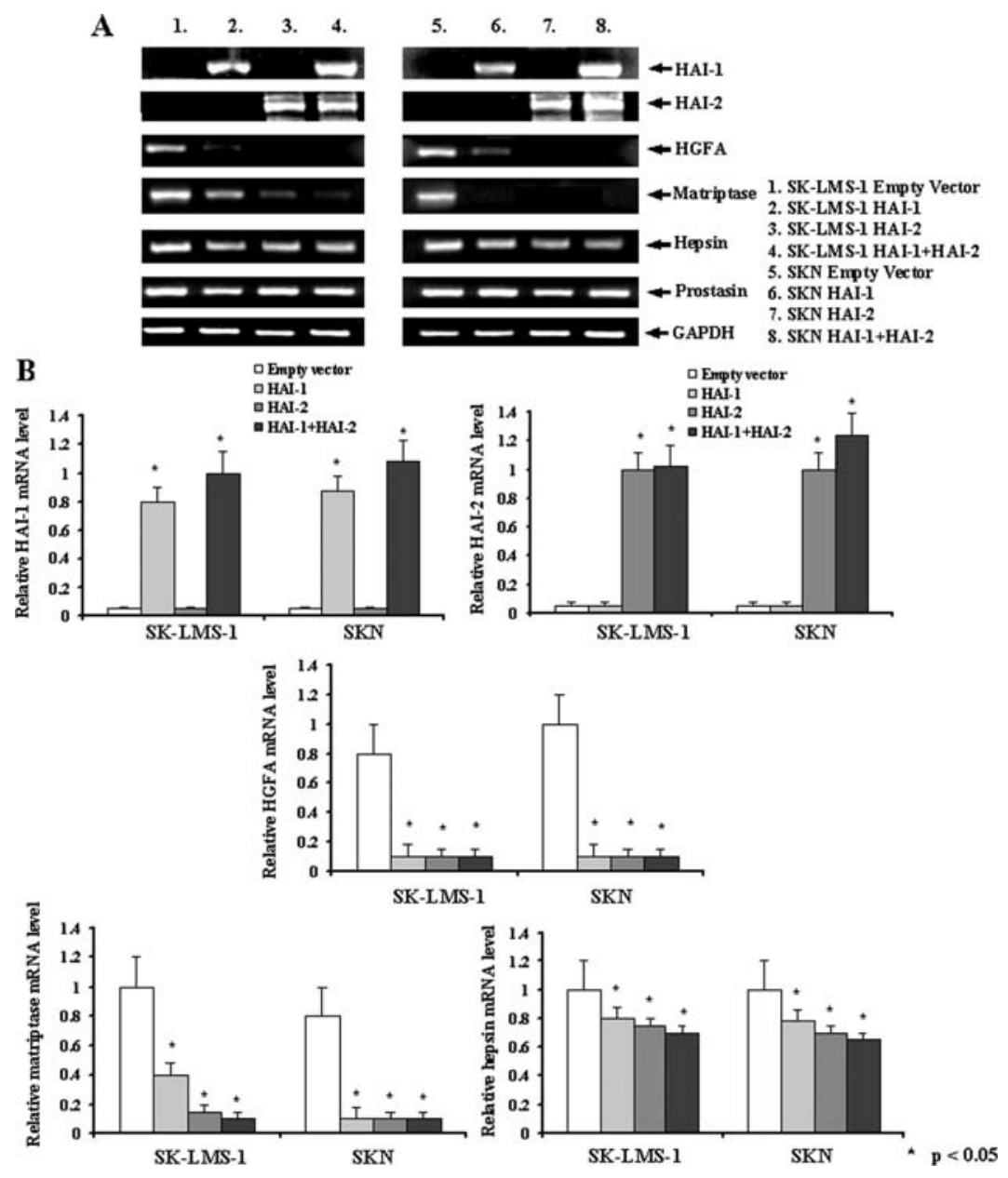

Figure 4. (A) PCR analysis of HAI-1, HAI-2, HGFA, matriptase, hepsin and prostasin expression levels after transient transfection with either HAI-1 vector or HAI-2 vector, or both HAI-1 vector and HAI-2 vector into SK-LMS-1 and SKN cells for $48 \mathrm{~h}$. GAPDH was used as the loading control. (B) Transient transfection with either HAI-1 vector or HAI-2 vector, or both HAI-1 vector and HAI-2 vector into SK-LMS-1 and SKN cells were analyzed for HAI-1, HAI-2, HGFA, matriptase and hepsin expressions using quantitative PCR. GAPDH was used as loading control. The results represent the mean \pm SD of triplicate independent experiments $\left({ }^{*} \mathrm{p}<0.05\right)$.

SK-LMS-1
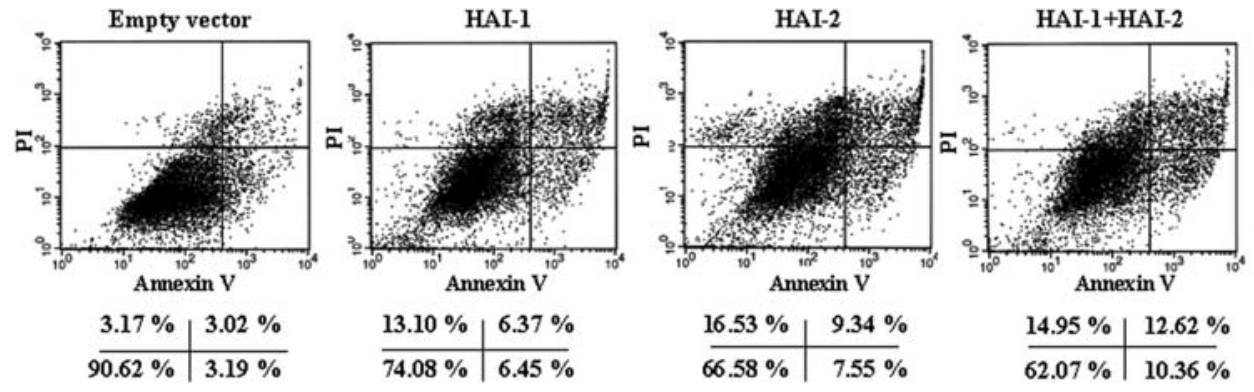

SKN
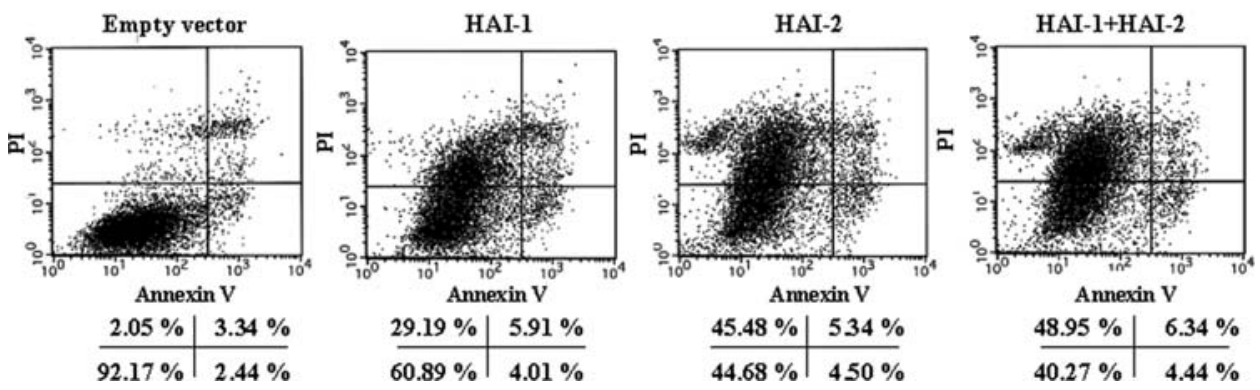

Figure 5. Representative flow cytometric data of apoptosis and necrosis in SK-LMS-1 and SKN cells transiently transfected for $48 \mathrm{~h}$ with either HAI-1 vector or HAI-2 vector, or both HAI-1 vector and HAI-2 vector. 

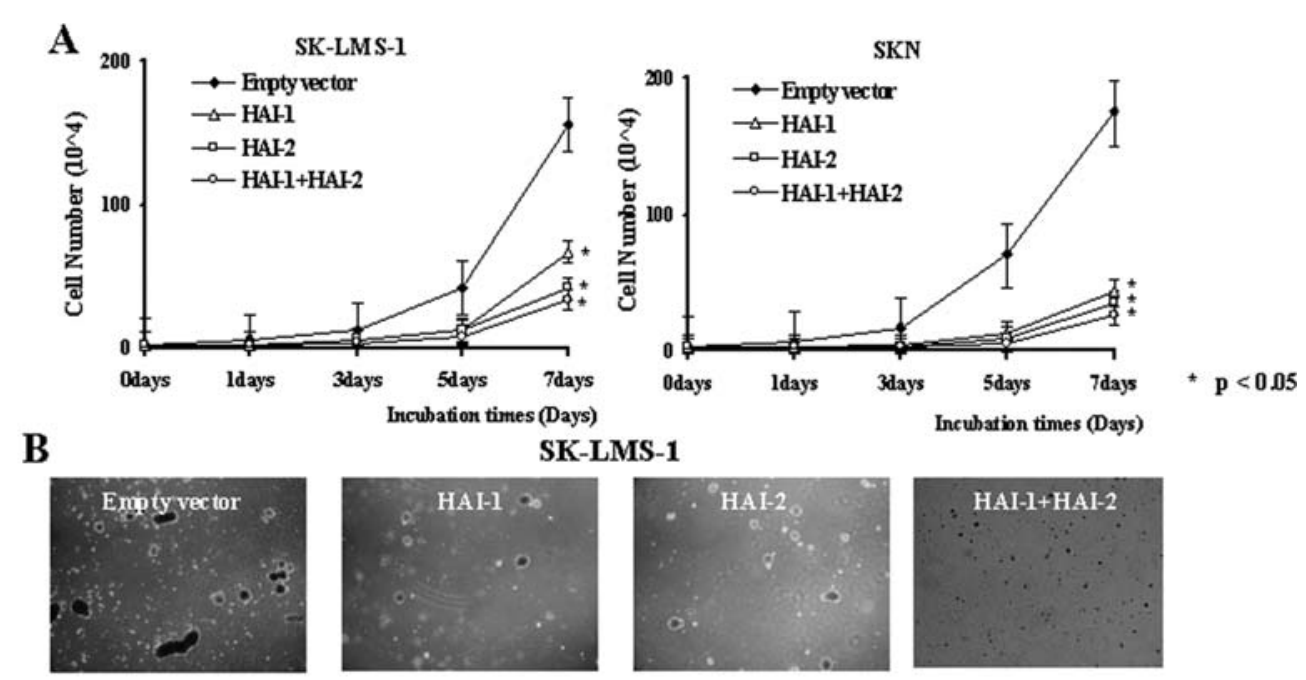

SK-LMS-1
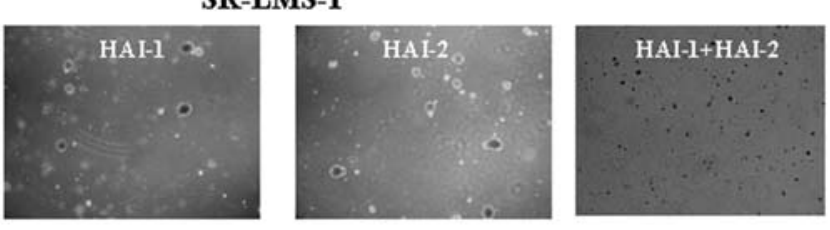

SKN
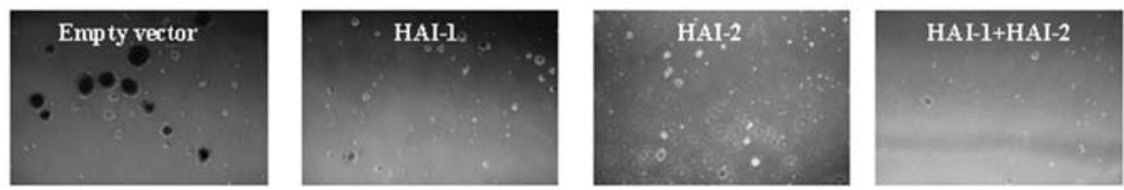

C Colony formation on soft agar of tranfected with either HAI-1 or HAI-2, or both HAI-1 and HAI-2

\begin{tabular}{clcc}
\multicolumn{3}{c}{ into SK-LMS-1 and SKN cell lines } \\
\hline Cell line & & Colonies/dish & $\begin{array}{c}\text { Inhibitory effect (\%) } \\
(\text { mean/SD) }\end{array}$ \\
\hline \multirow{2}{*}{ SK-LMS-1 } & Empty Vector & $10.8 \pm 2.8$ & 0 \\
& HAI-1 & $3.8 \pm 1.1$ & $64.8 \%^{*}$ \\
& HAI-2 & $2.7 \pm 0.9$ & $75.0 \%^{*}$ \\
& HAI-1+HAI-2 & $2.0 \pm 0.9$ & $81.5 \%^{*}$ \\
\hline SKN & Enpty Vector & $223.5 \pm 43.7$ & 0 \\
& HAI-1 & $503 \pm 10.2$ & $77.5 \%^{*}$ \\
& HAI-2 & $34.6 \pm 9.2$ & $84.5 \%^{*}$ \\
\hline HAI-1+HAI-2 & $21.1 \pm 8.8$ & $90.1 \%^{*}$ \\
\hline${ }^{2}$ Numbers represent data from triplicate experiments & ${ }^{*} \mathrm{p}<0.01$
\end{tabular}

Figure 6. Effects on the growth of SK-LMS-1 and SKN cells transiently transfected for $48 \mathrm{~h}$ with either HAI-1 vector or HAI-2 vector, or both HAI-1 vector and HAI-2 vector. (A) Monolayer growth of SK-LMS-1 and SKN cells transiently transfected with either HAI-1 vector or HAI-2 vector, or both HAI-1 vector and HAI-2 vector incubated for 1, 3, 5 and 7 days in DMEM or Ham's F12 medium supplemented with 10\% FBS. (B) Colony formation on cell aggregation of SK-LMS-1 and SKN cells after transient transfection with either HAI-1 vector or HAI-2 vector, or both HAI-1 vector and HAI-2 vector. (C) Colony formation on soft agar of SK-LMS-1 and SKN cells after transient transfection with either HAI-1 vector or HAI-2 vector, or both HAI-1 vector and HAI-2 vector. The data from triplicate experiments are shown.

cancer cell migration and invasion (44). Prostate cancer cells, after loss of HAI-1, showed an increase in invasiveness and cellular motility in vitro (45). Engineered overexpression of HAI-1 in glioblastoma cells reduced the invasiveness in vitro (46). In this study, we used MTS assays, cell viability, motility invasiveness assays, matrigel invasion assays, FITCconjugated Annexin V assays, and cell growth assays to examine the effects on SK-LMS-1 and SKN cells after transient transfection with either HAI-1 or HAI-2, or both HAI- 1 and HAI-2. Both HAI-1 and HAI-2 significantly inhibited cell proliferation, migration and cellular invasion. Interestingly, HAI-1 and HAI-2 induced apoptosis and necrosis in SK-LMS-1 and SKN cells, as shown in Fig. 5. Overall, HAI-1 and HAI-2 mediated cell proliferation, migration and cellular invasion which resulted in apoptosis and necrosis through a reduction of HGFA, hepsin, and matriptase expression. HAI-2 is a more efficient inhibitor than HAI-1. Furthermore, transient transfection of both HAI-1 and HAI-2 significantly reduced tumorigenesis as compared with HAI-2 alone. These findings indicate that HAI-1 and HAI-2 may be possible tumor suppressor genes of uterine LMS.

In conclusion, this study revealed a critical role for HAI-1 and HAI-2 in the disruption of uterine LMS. These findings indicate that HAI-1 and HAI-2 could be therapeutic agents for the treatment of uterine LMS.

\section{Acknowledgments}

We are grateful to Dr H. Kataoka for providing the HAI-1 and HAI-2 proteins to the Department of Section of Oncopathology and Regenerative Biology, Department of Pathology, Faculty of Medicine, University of Miyazaki, Japan.

\section{References}

1. Harlow BL, Weiss NS and Lofton S: The epidemiology of sarcomas of the uterus. J Natl Cancer Inst 76: 399-402, 1986. 
2. Arrastia CD, Fruchter RG, Clark M, Maiman M, Remy JC, Macasaet M, Gates EJ, Di Maio T and Marzec T: Uterine carcinosarcomas: incidence and trends in management and survival. Gynecol Oncol 65: 158-163, 1997.

3. Pautier P, Genestie C, Rey A, Morice P, Roche B, Lhommé C, Haie-Meder C and Duvillard P: Analysis of clinicopathologic prognostic factors for 157 uterine sarcomas and evaluation of a grading score validated for soft tissue sarcoma. Cancer 88: $1425-1431,2000$.

4. Mayerhofer K, Obermair A, Windbichler G, Petru E, Kaider A, Hefler L, Czerwenka K, Leodolter S and Kainz C: Leiomyosarcoma of the uterus: a clinicopathologic multicenter study of 71 cases. Gynecol Oncol 74: 196-201, 1999.

5. Berchuck A, Rubin SC, Hoskins WJ, Saigo PE, Pierce VK and Lewis JL Jr: Treatment of uterine leiomyosarcoma. Obstet Gynecol 71: 845-850, 1988

6. Major FJ, Blessing JA, Silverberg SG, Morrow CP, Creasman WT, Currie JL, Yordan E and Brady MF: Prognostic factors in earlystage uterine sarcoma. A Gynecologic Oncology Group study. Cancer 71: 1702-1709, 1993.

7. Nordal RR, Kristensen GB, Kaern J, Stenwig AE, Pettersen EO and Tropé CG: The prognostic significance of stage, tumor size, cellular atypia, and DNA ploidy in uterine leiomyosarcoma. Acta Oncol 34: 797-802, 1995

8. Gadducci A, Sartori E, Landoni F, Zola P, Maggino T, Urgesi A. Lissoni A, Losa G and Fanucchi A: Endometrial stromal sarcoma: analysis of treatment failures and survival. Gynecol Oncol 63: 247-253, 1996

9. Dinh TA, Oliva EA, Fuller AF Jr, Lee H and Goodman A: The treatment of uterine leiomyosarcoma. Results from a 10-year experience (1990-1999) at the Massachusetts General Hospital. Gynecol Oncol 92: 648-652, 2004.

10. Dinh TV and Woodruff JD: Leiomyosarcoma of the uterus. Am J Obstet Gynecol 144: 817-823, 1982.

11. Jeffers M, Rong S and Vande Woude GF: Enhanced tumorigenicity and invasion-metastasis by hepatocyte growth factor/ scatter factor-met signaling in human cells concomitant with induction of the urokinase proteolysis network. Mol Cell Biol 16: 1115-1125, 1996.

12. Comoglio PM: Structure, biosynthesis and biochemical properties of the HGF receptor in normal and malignant cells. EXS 65: $131-165,1993$

13. Rosen EM, Nigam SK and Goldberg ID: Scatter factor and the c-Met receptor: a paradigm for mesenchymal/epithelial interactions. J Cell Biol 127: 1783-1787, 1994.

14. Rong S, Jeffers M, Resau JH, Tsarfaty I, Oskarsson M and Vande Woude GF: Met expression and sarcoma tumorigenicity. Cancer Res 53: 5355-5360, 1993.

15. Shimomura T, Denda K, Kitamura A, Kawaguchi T, Kito M, Kondo J, Kagaya S, Qin L, Takata H, Miyazawa K and Kitamura N: Hepatocyte growth factor activator inhibitor, a novel Kunitz-type serine protease inhibitor. J Biol Chem 272: 6370-6376, 1997.

16. Kawaguchi T, Qin L, Shimomura T, Kondo J, Matsumoto K, Denda K and Kitamura N: Purification and cloning of hepatocyte growth factor activator inhibitor type 2, a Kunitz-type serine protease inhibitor. J Biol Chem 272: 27558-27564, 1997.

17. Kataoka H, Itoh H, Nuki Y, Hamasuna R, Naganuma $S$, Kitamura $\mathrm{N}$ and Shimomura T: Mouse hepatocyte growth factor (HGF) activator inhibitor type 2 lacking the first Kunitz domain potently inhibits the HGF activator. Biochem Biophys Res Commun 290: 1096-1100, 2002.

18. Müller-Pillasch F, Wallrapp C, Bartels K, Varga G, Friess H, Büchler M, Adler G and Gress TM: Cloning of a new Kunitztype protease inhibitor with a putative transmembrance domain overexpressed in pancreatic cancer. Biochim Biophys Acta 1395: $88-95,1998$.

19. Kirchhofer D, Peek M, Lipari MT, Billeci K, Fan B and Moran P: Hepsin activates pro-hepatocyte growth factor and is inhibited by hepatocyte growth factor activator inhibitor-1B (HAI-1B) and HAI-2. FEBS Lett 579: 1945-1950, 2005.

20. Kataoka H, Miyata S, Uchinokura S and Itoh H: Roles of hepatocyte growth factor (HGF) activator and HGF activator inhibitor in the pericellular activation of $\mathrm{HGF} / \mathrm{scatter}$ factor. Cancer Metastasis Rev 22: 223-236, 2003.

21. Fan B, Wu TD, Li W and Kirchhofer D: Identification of hepatocyte growth factor activator inhibitor-1B as a potential physiological inhibitor of prostasin. J Biol Chem 280: 34513-34520, 2005 .
22. Kataoka $\mathrm{H}$, Hamasuna $\mathrm{R}$, Itoh $\mathrm{H}$, Kitamura $\mathrm{N}$ and Koono $\mathrm{M}$ : Activation by hepatocyte growth factor/scatter in colorectal carcinoma. Cancer Res 60: 6148-6159, 2000.

23. Delaria KA, Muller DK, Marlor CW, Brown JE, Das RC, Roczniak SO and Tamburini PP: Characterization of placental bikunin, a novel human serine protease inhibitor. J Biol Chem 272: 12209-12214, 1997.

24. Nakamura K, Abarzua F, Hongo A, Kodama J, Nasu Y, Kumon H and Hiramatsu Y: Hepatocyte growth factor activator inhibitor-2 (HAI-2) is a favorable prognosis marker and inhibits cell growth through the apoptotic pathway in cervical cancer. Ann Oncol 20: 63-70, 2009

25. Nakamura K, Abarzua F, Kodama J, Hongo A, Nasu Y, Kumon H and Hiramatsu Y: Expression of hepatocyte growth factor activator inhibitors (HAI-1 and HAI-2) in ovarian cancer. Int J Oncol 34: 345-353, 2009.

26. Nakamura K, Abarzua F, Hongo A, Kodama J, Nasu Y, Kumon $\mathrm{H}$ and Hiramatsu Y: The role of hepatocyte growth factor activator inhibitor-1 (HAI-1) as a prognostic indicator in cervical cancer. Int J Oncol 35: 239-248, 2009.

27. Saleem M, Adhami VM, Zhong W, Longley BJ, Lin CY, Dickson RB, Reagan-Shaw S, Jarrard DF and Mukhtar H: A novel biomarker for staging human prostate adenocarcinoma: overexpression of matriptase with concomitant loss its inhibitor, hepatocyte growth factor activator inhibitor-1. Cancer Epidemiol Biomarkers Prev 15: 217-227, 2006.

28. Parr C, Watkins G, Mansel RE and Jiang WG: The hepatocyte growth factor regulatory factors in human breast cancer. Clin Cancer Res 10: 202-211, 2004.

29. Zeng L, Cao J and Zhang X: Expression of serine protease SNC19/matriptase and its inhibitor hepatocyte growth factor activator inhibitor type 1 in normal and malignant tissues of gastrointestinal tract. World J Gastroenterol 11: 6202-6207, 2005.

30. Hamasuna R, Kataoka H, Meng JY, Itoh H, Moriyama T, Wakisaka S and Koono M: Reduced expression of hepatocyte growth factor activator inhibitor type-2/placental bikunin (HAI-2/ PB) in human glioblastomas: implication for anti-invasive role of HAI-2/PB in glioblastoma cells. Int J Cancer 93: 339-345, 2001.

31. Fukai K, Yokosuka O, Chiba T, Hirasawa Y, Tada M, Imazeki F, Kataoka $\mathrm{H}$ and Saisho H: Hepatocyte growth factor activator inhibitor 2/placental bikunin (HAI-2/PB) gene is frequently hypermethylated in human hepatocellular carcinoma. Cancer Res 63: 8674-8679, 2003.

32. Betsunoh H, Mukai S, Akiyama Y, Fukushima T, Minamiguchi N, Hasui Y, Osada Y and Kataoka H: Clinical relevance of hepsin and hepatocyte growth factor activator inhibitor type 2 expression in renal cell carcinoma. Cancer Sci 98: 491-498, 2007.

33. Kataoka H, Shimomura T, Kawaguchi T, Hamasuna R, Itoh H, Kitamura N, Miyazawa K and Koono M: Hepatocyte growth factor activator inhibitor type 1 is a specific cell surface binding protein of hepatocyte growth factor activator (HGFA) and regulates HGFA activity in the pericellular microenvironment. J Biol Chem 275: 40453-40462, 2000.

34. Nakamura K, Nasu Y, Hongo A, Matsuo T, Kodama J, Ebara S, Nagai A, Abrzua F, Kumon H and Hiramatsu Y: Hepsin shows inhibitory effects through apoptotic pathway on ovarian cancer cell lines. Int J Oncol 28: 393-398, 2006.

35. Cheng H, Fukushima T, Takahashi N, Tanaka H and Kataoka H: Hepatocyte growth factor activator inhibitor type 1 regulates epithelial to mesenchymal transition through membrane-bound serine proteinases. Cancer Res 69: 1828-1835, 2009

36. Sakashita K, Mimori K, Tanaka F, Tahara K, Inoue H, Sawada T, Ohira M, Hirakawa K and Mori M: Clinical significance of low expression of Prostasin mRNA in human gastric cancer. J Surg Oncol 98: 559-564, 2008.

37. Nagakawa O, Yamagishi T, Fujiuchi Y, Junicho A, Akashi T, Nagaike $\mathrm{K}$ and Fuse H: Serum hepatocyte growth factor activator (HGFA) in benign prostatic hyperplasia and prostate cancer. Eur Urol 48: 686-690, 2005

38. Szabo R, Hobson JP, List K, Molinolo A, Lin CY and Bugge TH: Potent inhibition and global co-localization implicate the transmembrane Kunitz-type serine protease inhibitor hepatocyte growth factor activator inhibitor-2 in the regulation of epithelial matriptase activity. J Biol Chem 283: 29495-29504, 2008

39. Netzel-Arnett S, Hooper JD, Szabo R, Madison EL, Quigley JP, Bugge TH and Antalis TM: Membrane anchored serine proteases: a rapidly expanding group of cell surface proteolytic enzymes with potential roles in cancer. Cancer Metastasis Rev 22: $237-258,2003$ 
40. Lee SL, Dickson RB and Lin CY: Activation of hepatocyte growth factor and urokinase/plasminogen activator by matriptase, an epithelial membrane serine protease. J Biol Chem 275: 36720-36725, 2000

41. Moran P, Li W, Fan B, Vij R, Eigenbrot C and Kirchhofer D: Pro-urokinase-type plasminogen activator is a substrate for hepsin. J Biol Chem 281: 30439-30446, 2006.

42. Takeuchi T, Harris JL, Huang W, Yan KW, Coughlin SR and Crai CS: Cellular localization of membrane-type serine protease 1 and identification of protease-activated receptor-2 and single-chain urokinase-type plasminogen activator as substrates. J Biol Chem 275: 26333-26342, 2000.

43. Netzel-Arnett S, Currie BM, Szabo R, Lin CY, Chen LM, Chai KX, Antalis TM, Bugge TH and List K: Evidence for a matriptase-prostasin proteolytic cascade regulating terminal epidermal differentiation. J Biol Chem 281: 32941-32945, 2006.
44. Parr C and Jiang WG: Hepatocyte growth factor activation inhibitors (HAI-1 and HAI-2) regulate $\mathrm{HGF}$-induced invasion of human breast cancer cells. Int J Cancer 119: 1176-1183, 2006.

45. Sanders AJ, Parr C, Mason MD and Jiang WG: Suppression of hepatocyte growth factor activator inhibitor-1 leads to a more aggressive phenotype of prostate cancer cells in vitro. Int $\mathrm{J} \mathrm{Mol}$ Med 20: 613-619, 2007

46. Miyata S, Fukushima T, Kohama K, Tanaka H, Takeshima H and Kataoka H: Roles of Kunitz domains in the anti-invasive effect of hepatocyte growth factor activator inhibitor type 1 in human glioblastoma cells. Hum Cell 20: 100-106, 2007. 